\title{
Anticancer Effect of Medicinal Mushroom with Prooxidant Activity on Human Bladder Cancer Cells
}

\author{
Cristina Fox, Roger Yau, Muhammad Choudhury, John Phillips, Sensuke Konno* \\ Department of Urology, New York Medical College, Valhalla, New York, USA \\ *Corresponding author: sensuke_konno@nymc.edu
}

\begin{abstract}
Oxidative stress (OXS) has been recently considered as one of anticancer strategies by taking advantage of higher vulnerability of cancer cells (than normal cells) to OXS. In fact, the successful outcomes using OXS have been reported in several cancer cases. A medicinal mushroom extract, PE isolated from Poria mushroom, has been shown to have anticancer/antitumor activity, although its anticancer mechanism has not been fully understood but may involve OXS. We investigated if PE might have anticancer effect on human bladder cancer cells through OXS in vitro. A dose-dependent ( $0-200 \mu \mathrm{g} / \mathrm{ml}$ of PE) study was first performed to assess cell viability using MTT assay. $\mathrm{PE}$ led to a significant reduction in cell viability with the $\mathrm{IC}_{50}(50 \%$ inhibitory concentration) of $100 \mu \mathrm{g} / \mathrm{ml}$. A possible anticancer role of OXS was then assessed by lipid peroxidation (LPO) assay. The results indicated that PE indeed exerted $\sim 2.1$-fold greater OXS (than controls) on the cells. The anticancer mechanism of PE was further explored, focusing on glycolysis, metabolic signaling pathways, and apoptosis. Two glycolytic parameters, hexokinase (HK) activity and cellular ATP level, have significantly declined, suggesting the inhibition of glycolysis. Coupled with the reduced ATP level, AMP-activated protein kinase (AMPK) was activated, while protein kinase B (Akt) was inactivated and concomitantly mammalian target of rapamycin (mTOR) was inhibited. These results imply the growth cessation, followed by cell death. Western blot analysis also revealed that such cell death was more likely linked to apoptosis, indicated by the bcl-2 down-regulation and the Bax up-regulation. Therefore, PE is a natural anticancer agent with prooxidant activity exerting OXS, which leads to inhibition of glycolysis, modulations of metabolic signaling pathways, and ultimately apoptosis. It may have clinical implications in oral and/or intravesical administration for a safer and better therapeutic option for bladder cancer.
\end{abstract}

Keywords: anticancer, oxidative stress, mushroom, prooxidant, bladder cancer

Cite This Article: Cristina Fox, Roger Yau, Muhammad Choudhury, John Phillips, and Sensuke Konno, "Anticancer Effect of Medicinal Mushroom with Prooxidant Activity on Human Bladder Cancer Cells." Journal of Cancer Research and Treatment, vol. 6, no. 2 (2018): 54-59. doi: 10.12691/jcrt-6-2-5.

\section{Introduction}

Bladder cancer is the second common urologic malignancy next to prostate cancer in the United States. Approximately 79,000 new cases and nearly 17,000 deaths of patients with bladder cancer are estimated this year (2017) [1]. In males, bladder cancer represents the fourth most frequent diagnosis of new cancer annually [1]. Currently, transitional cell carcinoma (TCC) is the most prevalent type ( $\sim 90 \%)$ of bladder cancers and approximately $80 \%$ of TCC present superficial bladder tumor while $15 \%$ are invasive and $5 \%$ are metastatic disease [2]. Unfortunately, regardless of non-muscle invasive or muscle invasive cases, the current treatment options are limited. For non-muscle invasive bladder cancer, endoscopic transurethral resection (TUR) is the primary modality with or without intravesical chemotherapy or immunotherapy. However, $60 \%-70 \%$ of patients will recur and $\sim 25 \%$ will progress to muscle invasive disease within 5 years [3], becoming untreatable and fatal. For muscle invasive disease, chemotherapy and surgery are the two major modalities but the prognosis is less satisfactory because only $60 \%$ of these patients will survive five years later, due to distant recurrence [4]. Hence, it is crucial to establish the improved modalities to prevent multiple recurrences and progression to an advanced invasive disease. However, over the past several decades, only limited improvement or progress has been made. There is the urgent need for a more effective, safer treatment modality with few side effects.

Recently, the medicinal aspects of various natural agents and substances have gained more public attention. Those include herbs, mushrooms, flowers, fruits, plant seeds, sea weeds, algae, tea, bark, shark cartilage and so on. We were particularly interested in one well-established medicinal mushroom called Poria mushroom and its bioactive extract, PE. This mushroom has been used in Traditional Chinese Medicine for 2,000 years [5] and found to have various properties, such as antioxidant, anticancer/antitumor, immunomodulatory, renoprotective effects etc. $[6,7,8]$. As $\mathrm{PE}$ is a natural agent, it is conceivable that it may have few side effects and clinical implications in cancer treatment.

Oxidative stress (OXS), i.e. generation of reactive oxygen species (ROS), has been known to exert adverse effects on a variety of normal and cancer cells, injuring, damaging and even killing them [9]. ROS include superoxide 
anion, singlet oxygen, hydroxyl radicals, hydrogen peroxide etc., which are commonly generated through incomplete reduction of molecular oxygen during aerobic metabolic processes [10]. Although OXS has never been appreciated by any means, it has been recently considered as one of anticancer strategies by taking advantage of cancer cells being more vulnerable to OXS than normal counterparts [11]. The exact reason for such higher vulnerability of cancer cells remains elusive, but at least it is believed to be the inherent difference in tissue-specific antioxidant system, i.e. the weakened or lack of antioxidant enzymes in cancer cells. As a matter of fact, the successful outcomes using OXS have been reported in several cancer cases $[11,12,13]$.

Taking this OXS strategy into consideration, it was of our interest to study if PE could exert OXS on bladder cancer cells because PE may have not only antioxidant activity but also "prooxidant" activity (exerting OXS) like vitamin C [14]. If PE indeed exerts OXS, it could be the primary factor for its anticancer activity that has been demonstrated [6]. We also explored the OXS-mediated anticancer mechanism of PE, focusing on glycolysis, the status of metabolic signaling pathways, and possible induction of apoptosis. More details and interesting findings are described and discussed herein.

\section{Materials and Methods}

\subsection{Cell Culture}

Human bladder cancer 5637 cells, obtained from the American Type Culture Collection (ATCC; Manassas, VA), were cultured in RPMI 1640 medium supplemented with $10 \%$ fetal bovine serum, penicillin $(100 \mathrm{U} / \mathrm{ml})$, and streptomycin $(100 \mu \mathrm{g} / \mathrm{ml})$. Routinely, culture medium was changed every 3 to 4 days and the passage of cells was performed weekly. PE, the bioactive extract of Poria mushroom, was a generous gift from the manufacturer (Mushroom Wisdom, Inc., East Rutherford, NJ). For experiments, cells were seeded in the 6-well culture plate at the initial cell density of $2 \times 10^{5}$ cells $/ \mathrm{ml}$ and cultured with varying concentrations of PE. Cell viability was determined at $72 \mathrm{~h}$ by MTT assay.

\subsection{MTT Assay (Cell Viability Test)}

Cell viability was determined by the MTT (3-[4,5dimethylthiazol-2-yl]-2,5-diphenyl-tetrazolium bromide) assay following the vendor's protocol (Sigma-Aldrich, St. Louis, MO). At the harvest time, MTT reagent $(1 \mathrm{mg} / \mathrm{ml})$ was added to each well in the 6-well plate, followed by 3-h incubation at $37^{\circ} \mathrm{C}$. After discarding MTT reagent, dimethyl sulfoxide $(1 \mathrm{ml}$ each) was added to the plate to dissolve formazan precipitates formed. Formazan solution (purple) was read at $595 \mathrm{~nm}$ on a microplate reader. Cell viability was then expressed by the percent (\%) of viable cells relative to the control reading $(100 \%)$.

\subsection{Lipid Peroxidation (LPO) Assay}

The severity of oxidative stress can be assessed by LPO assay, which measures the amount of malondialdehyde
(MDA) formed, due to damage in the plasma membrane under oxidative stress [15]. This indicates - the more MDA formed, the greater oxidative stress. The detailed procedures were described in the vendor's protocol (ABCAM, Cambridge, MA). The amount of MDA formed was determined from the MDA standards and expressed by $\mu \mathrm{M}$.

\subsection{Assays for Glycolytic Parameters}

To assess possible effects of PE on glycolysis, two glycolytic parameters, hexokinase (HK) activity and cellular ATP level, were determined using the HK or ATP Colorimetric Assay Kits (BioVision, Milpitas, CA), respectively, following the manufacturer's protocols.

HK Assay: Control or agents-treated cells were lysed in HK buffer provided and supernatant (cell lysates) was obtained by centrifugation. The NADH standards and cell lysates (20 $\mu \mathrm{g}$ per sample) were prepared in the $96-$ well plate and the reaction was started by the addition of reaction mix (containing substrate). Immediately the plate was placed in a microplate reader and the absorbance (OD) changes with time were monitored at $450 \mathrm{~nm}$ for $20 \mathrm{~min}$ with 5-min intervals. All readings were calculated and normalized and then HK activity was expressed by the \% of sample activity relative to the controls (100\%).

ATP Assay: Cells (2 × $10^{5}$ cells $\left./ \mathrm{ml}\right)$ in the 6-well plate were first lysed in ATP assay buffer and cell lysates were deproteinized with $\mathrm{HClO}_{4}$ (perchloric acid) and neutralized with $\mathrm{KOH}$ (potassium hydroxide). The ATP standards and samples ( $50 \mu \mathrm{l}$ per sample) were prepared in the 96-well plate and the reaction was started by the addition of reaction mixture. The plate was incubated at room temperature for $30 \mathrm{~min}$ in the dark. Absorbance (OD) at $570 \mathrm{~nm}$ was read on a microplate reader and ATP content was calculated by applying the sample readings to the ATP standards. The ATP level was then expressed by the $\%$ of sample readings relative to the controls $(100 \%)$.

\subsection{Western Blot Analysis}

An equal amount of cell lysates $(10 \mu \mathrm{g})$, obtained from control and agent-treated cells, was first subjected to $10 \%$ SDS gel electrophoresis and transferred to a nitrocellulose membrane (blot). The blot was first incubated for $90 \mathrm{~min}$ with the primary antibodies against three metabolic regulators, p-AMPK, p-Akt, and p-mTOR (Santa Cruz Biotechnology, Santa Cruz, CA), or with two apoptotic regulators, bcl-2 and Bax (Santa Cruz Biotechnology). After removing the primary antibodies, the blot was incubated with the secondary antibody conjugates for 30 $\mathrm{min}$. The specific immunoreactive protein bands were then detected by chemiluminescence following the manufacturer's protocol (Kirkegaard and Perry Laboratories, Gaithersberg, $\mathrm{MD})$.

\subsection{Statistical Analysis}

All data were presented as mean $\pm \mathrm{SD}$ (standard deviation), and statistical differences between groups are assessed with either the unpaired Student's $t$ test or oneway ANOVA analysis. Values of $p<0.05$ are considered to indicate statistical significance. 


\section{Results}

\subsection{Effects of PE on Cell Viability of Bladder Cancer 5637 Cells}

To assess anticancer effect of PE on 5637 cells, cell viability, i.e. the $\%$ of viable cells, was determined after they were treated with varying concentrations $(0-200 \mu \mathrm{g} / \mathrm{ml})$ of PE for $72 \mathrm{~h}$ using MTT assay. Although PE at $50 \mu \mathrm{g} / \mathrm{ml}$ had little effect, it led to a $\sim 50 \%$ and $\sim 90 \%$ reduction in cell viability at 100 and $200 \mu \mathrm{g} / \mathrm{ml}$, respectively, in a dose-dependent manner (Figure 1). Thus, PE appears to have anticancer effect, capable of significantly reducing 5637 cell viability. As PE $(100 \mu \mathrm{g} / \mathrm{ml})$ is proximate to its $\mathrm{IC}_{50}(50 \%$ inhibitory concentration), this concentration was then used in the rest of our study.

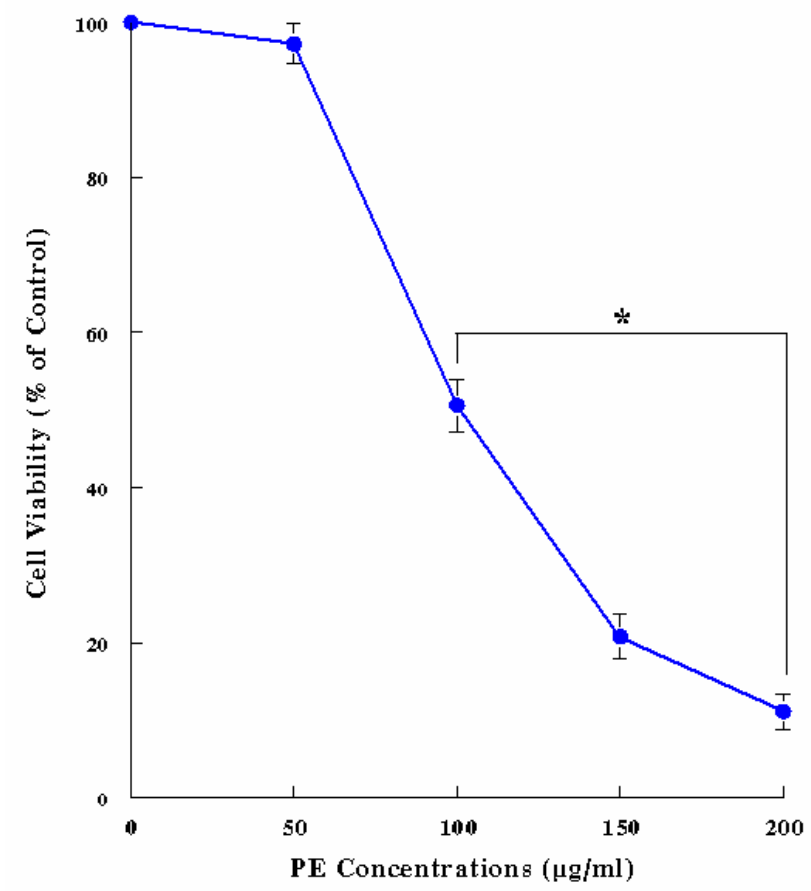

Figure 1. Dose-dependent effect of PE on cell viability. 5637 cells were cultured with varying concentrations of PE $(0-200 \mu \mathrm{g} / \mathrm{ml})$ and cell viability was assessed in $72 \mathrm{~h}$ by MTT assay. Cell viability was expressed by the percent (\%) of viable cells relative to controls (100\%). The data are mean \pm SD (standard deviation) from three separate experiments $\left({ }^{*} p<0.05\right.$ compared with control)

\subsection{Exertion of Oxidative Stress (OXS) by PE}

Whether PE-induced cell viability reduction could be due to OXS was examined next. Hydrogen peroxide $\left(\mathrm{H}_{2} \mathrm{O}_{2}, 50 \mu \mathrm{M}\right)$, one of typical ROS, was also used as a positive control for exerting OXS on cells. As OXS usually takes place at the early phase, after cells were briefly exposed to $\mathrm{PE}(100 \mu \mathrm{g} / \mathrm{ml})$ or $\mathrm{H}_{2} \mathrm{O}_{2}(50 \mu \mathrm{M})$ for 3 or $6 \mathrm{~h}$, they were subjected to LPO assay to determine the severity of OXS. Compared to controls, $\mathrm{H}_{2} \mathrm{O}_{2}$ and PE led to a $~ 3.2$-fold and $\sim 2.1$-fold increase in the MDA amounts at 6 hours, respectively (Figure 2). Thus, PE (as well as $\mathrm{H}_{2} \mathrm{O}_{2}$ ) appears to be capable of exerting severe OXS on 5637 cells, demonstrating its prooxidant activity and presumably accounting for the significant cell viability reduction (Figure 1).

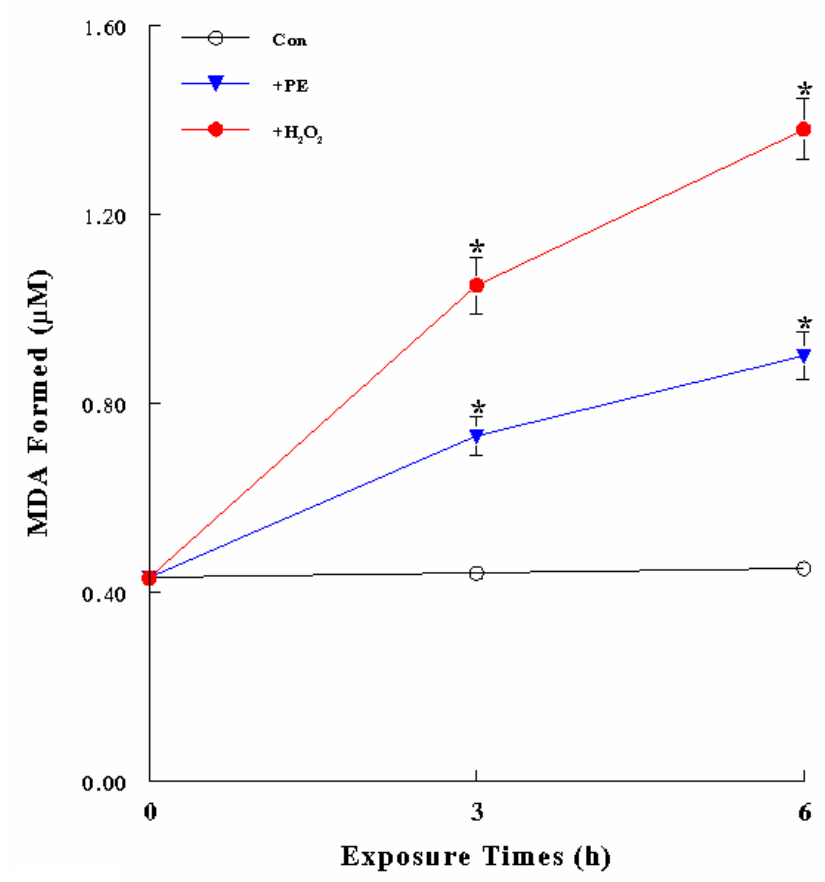

Figure 2. Assessment of severity of OXS. Cells were exposed to PE $(100 \mu \mathrm{g} / \mathrm{ml})$ or $\mathrm{H}_{2} \mathrm{O}_{2}(50 \mu \mathrm{M})$ for 3 or $6 \mathrm{~h}$, and the amount of MDA formed $(\mu \mathrm{M}))$ was assessed by LPO assay. All data are mean \pm SD from three independent experiments $(* p<0.05$ compared with controls)

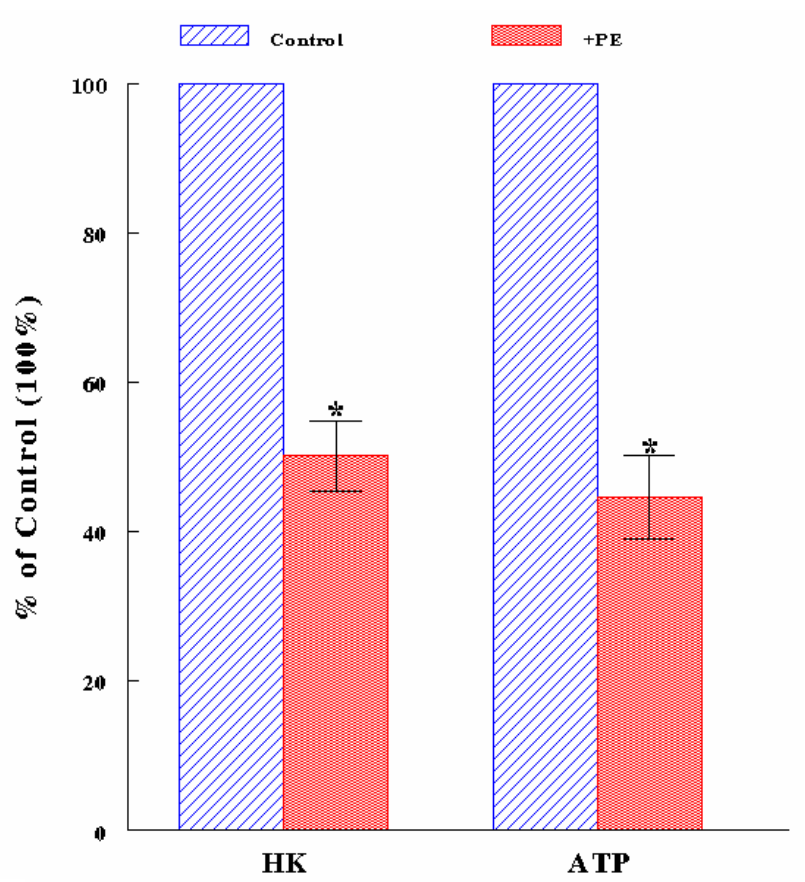

Figure 3. Inhibition of glycolysis by PE. Cells were treated with PE (100 $\mu \mathrm{g} / \mathrm{ml}$ ) for $72 \mathrm{~h}$ and subjected to HK and ATP assays separately. HK activity and ATP level are expressed by the \% relative to respective controls $(100 \%)$. The data are mean from three separate experiments $\left(^{*} p\right.$ $<0.05$ compared with control)

\subsection{Effect of PE-Exerted OXS on Glycolysis}

To have an insight into how PE-exerted OXS would induce a significant cell viability reduction or to understand the anticancer mechanism of PE, we first examined if glycolysis could be affected/inhibited by PE because it is the vital metabolic process required for cell proliferation and survival $[16,17]$. We specifically focused on one of 
key glycolytic enzymes, hexokinase (HK), which was involved in the irreversible committed step in glycolysis [18]. Assuming that HK was inhibited by PE, the amount of ATP produced or the cellular ATP level would also decline because the sequential glycolytic pathway could not be even initiated and completed. Accordingly, cells were treated with PE $(100 \mu \mathrm{g} / \mathrm{ml})$ for $72 \mathrm{~h}$ and $\mathrm{HK}$ activity and ATP level were assessed, as described in Materials and Methods. Compared to controls (100\%), HK activity declined to $\sim 50 \%$ (i.e. a $\sim 50 \%$ activity loss) with $\mathrm{PE}$ treatment (Figure 3). ATP assay revealed that the cellular ATP level was also down to $\sim 45 \%$ (i.e. a $\sim 55 \%$ reduction), due to HK inactivation. Thus, the reduction in HK and ATP levels by PE indicates the inhibition of glycolysis, eventually leading to the growth cessation and the cell viability reduction.

\subsection{Effects of OXS on Metabolic Signaling Pathways}

ATP (energy) reduction/depletion, due to the glycolysis inhibition, has been shown to affect metabolic signaling pathways, which play a pivotal role in cell proliferation and survival $[17,19]$. The cells treated with/without PE for $72 \mathrm{~h}$ were analyzed for three key regulators, AMP-activated protein kinase (AMPK) [20], serine/threonine protein kinase B (Akt) [21], or mammalian target of rapamycin (mTOR) [22] by Western blots. Such analysis revealed that the expression of AMPK was enhanced or phosphorylated (activated), whereas those of Akt and mTOR were reduced or dephosphorylated (inactivated) with $\mathrm{PE}$ treatment (Figure 4). Therefore, this activation of AMPK concomitant with inactivation of $\mathrm{Akt} / \mathrm{mTOR}$ by $\mathrm{PE}$ suggests that 5637 cells more likely undergo cell death as it has been shown in certain cancer cells $[20,21,22]$.

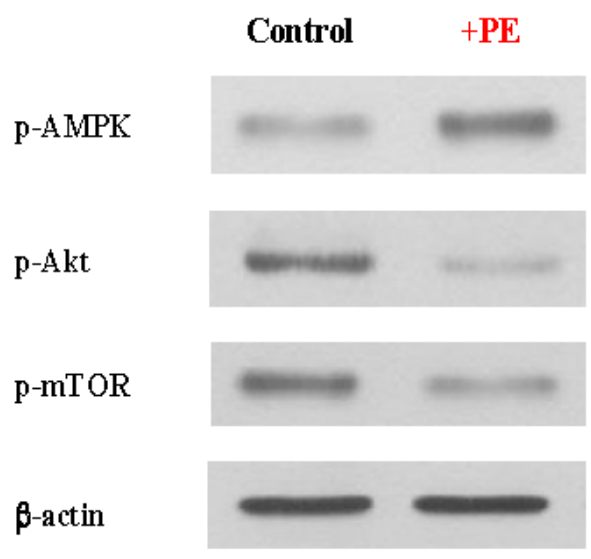

Figure 4. Modulations of metabolic regulators by PE. Cells treated with PE $(100 \mu \mathrm{g} / \mathrm{ml})$ for $72 \mathrm{~h}$ were analyzed for three key metabolic regulators using Western blots. Autoradiographs of p-AMPK, p-Akt, and p-mTOR expressed in control and PE-treated cells are shown for comparison. Beta-actin is also shown as a loading control

\subsection{Possible Induction of Apoptosis by PE}

As it seems that the cell viability reduction by PE could be primarily due to cell death, we next examined if such cell death would be linked to apoptosis. The cells treated with/without PE for $72 \mathrm{~h}$ were analyzed for two key apoptotic regulators by Western blots. Such analysis showed that PE treatment led to the down-regulation (reduced expression) of anti-apoptotic bcl-2 and the upregulation (elevated expression) of pro-apoptotic Bax (Figure 5). Such modulations of two regulators will favor and indicate induction of apoptosis [23]. It is thus plausible that PE-induced cell death would be rather linked to apoptosis.

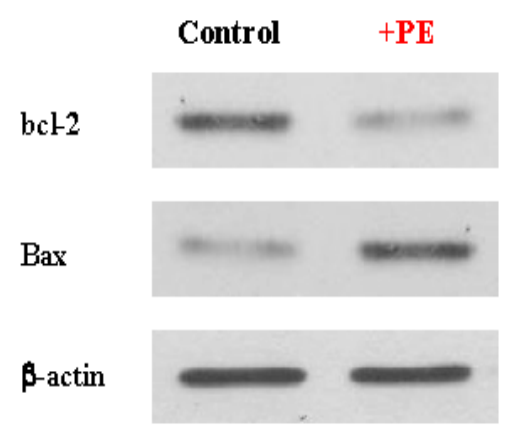

Figure 5. Induction of apoptosis. Autoradiographs of two apoptotic regulators, bcl-2 and Bax, expressed in control and PE-treated cells are shown following Western blot analysis

\section{Discussion}

Because of the ineffective therapeutic options and unsatisfactory outcomes, bladder cancer patients and their families are desperately seeking for a better, more effective therapeutic modality. We have been exploring an alternative or unconventional way to improve the therapeutic efficacy using natural substances or products. We have come across the bioactive mushroom extract, $\mathrm{PE}$, isolated from Poria mushroom and investigated if it might have anticancer effect on human bladder cancer cells.

PE had a significant anticancer effect with the $\mathrm{IC}_{50}$ of $\sim 100 \mu \mathrm{g} / \mathrm{ml}$ on bladder cancer 5637 cells (Figure 1). To explore its anticancer mechanism, we examined if it could be associated with oxidative stress (OXS). LPO assay showed that PE did exert severe OXS on 5637 cells as indicated by a $\sim 2$-fold increase in MDA formation (Figure 2). This finding suggests that anticancer mechanism of PE would be substantially attributed to OXS. It has been well known that OXS was harmful to both normal and cancer cells but particularly cancer cells were highly or more vulnerable to OXS [11]. Although the reason for such discrepancy remains to be elucidated, the inherent difference in tissue-specific antioxidant system is believed to at least in part account for it. Based on this uncanny nature of cancer cells, OXS is now considered as one of anticancer strategies. Suppose relatively weak OXS were exerted on normal and cancer cells, it could be yet severe or strong enough to kill cancer cells (due to weaker antioxidant enzymes) but not severe enough to even injure normal cells (with stronger enzymes). More studies on the status of antioxidant enzymes in bladder cancer need to be further conducted.

Nevertheless, to elucidate how PE-exerted OXS would lead to the cell viability reduction or even cell death, we examined its effect on glycolysis, an essential metabolic process. Such study showed that HK was specifically targeted and inactivated by OXS, shutting down the glycolytic pathway. As a result, ATP synthesis was 
also halted and its cellular level significantly declined. These results indicate the inhibition of glycolysis and cells may subsequently cease cell growth and result in cell death.

As it has been shown that ATP depletion (due to the glycolysis inhibition) would also influence metabolic signaling pathways [17], this possibility was tested. We found that the three key signaling regulators, AMPK, Akt, and mTOR [20,21,22], were modulated by PE (Figure 4). In fact, AMPK was highly phosphorylated (activated) while Akt was dephosphorylated (inactivated) by PE. Both AMPK and Akt must be phopsphrylated to become active and functional [20,21]. Activation of AMPK has been shown to be cytotoxic to a variety of cancer cells and promote apoptosis [24], while Akt activation would promote cell survival by inhibiting apoptosis [21,25]. Hence, AMPK and Akt will interact directly through two opposed mechanisms [20], and activation or inhibition of these two parameters would determine an anabolic or catabolic process. In addition, activation of AMPK and inhibition of Akt (as seen in this study) is known to inactivate the master orchestrator of cell proliferation, mTOR, which is a protein kinase working with Akt to promote protein synthesis and cell proliferation [22]. This is consistent with our finding that mTOR (along with Akt) was indeed dephosphorylated or inactivated by PE (Figure 4). Thus, AMPK activation and Akt/mTOR inactivation induced by PE would ultimately lead to cell death, more likely accounting for the cell viability reduction. Lastly, whether such cell death induced by PE would be linked to apoptosis was examined. Western blot analysis revealed the down-regulation of bcl-2 and the up-regulation of Bax with $\mathrm{PE}$ treatment (Figure 5). As bcl-2 is anti-apoptotic but Bax is pro-apoptotic [23], this finding is rather in support of apoptosis. Thus, PE-induced cell death appears to follow apoptotic pathway.

\section{Conclusion}

In this study, PE demonstrated its anticancer activity on human bladder cancer 5637 cells in vitro. Such anticancer activity appears to be primarily attributed to OXS, which leads to inhibition of glycolysis, modulation of metabolic signaling pathways, and ultimate induction of apoptosis. As a result, cell viability will significantly decline following PE treatment. Therefore, PE with prooxidant activity exerting OXS could be a promising natural anticancer agent that may offer a safer and more effective therapeutic option for bladder cancer.

\section{Conflict of Interest}

The authors have no competing interests.

\section{Acknowledgements}

The authors thank Mike Shirota (Mushroom Wisdom, Inc.) for generously providing us with $\mathrm{PE}$ and are indebted to financial support from Seize the Ribbon in this study.

\section{Abbreviations}

OXS: oxidative stress

PE: Poria mushroom extract

MTT: 3-[4,5-dimethylthiazol-2-yl]-2,5-diphenyl-

tetrazolium bromide

ROS: reactive oxygen species

LPO: lipid peroxidation

TCC: transitional cell carcinoma

TUR: transurethral resection

MDA: malondialdehyde

SDS: sodium dodecyl sulfate

HK: hexokinase

AMPK: AMP-activated protein kinase

Akt: serine/threonine protein kinase B

mTOR: mammalian target of rapamycin

$\mathrm{IC}_{50}: 50 \%$ inhibitory concentration

OD: optical density

ANOVA: analysis of variance

SD: standard deviation.

\section{References}

[1] Siegel RL, Miller KD, Jemal A. Cancer statistics 2017. CA Cancer J Clin. 2017; 67(1): 7-30.

[2] Pow-Sang JM, Seigne JD. Contemporary management of superficial bladder cancer. Cancer Control. 2000; 7(4): 335-339.

[3] Kaufman DS, Shipley WU, Feldman AS. Bladder cancer. Lancet. 2009; 374(9685): 239-249.

[4] Grossman HB, Natale RB, Tangen CM, et al. Neoadjuvant chemotherapy plus cystectomy compared with cystectomy alone for locally advanced bladder cancer. N Engl J Med. 2003; 349(9): 859-866.

[5] Rios JL. Chemical constituents and pharmacological properties of Poria cocos. Planta Med. 2011; 77(7): 681-691.

[6] Zhou L, Zhang Y, Gapter LA, Ling H, Agarwal R, Ng KY. Cytotoxic and antioxidant activities of lanostane-type triterpenes isolated from Poria cocos. Chem Pharm Bull (Tokyo). 2008; 56(10): 1459-1462.

[7] Chen X, Zhang L, Cheung PC. Immunopotentiation and antitumor activity of carboxymethylated-sulfated $\beta$-(1 $\rightarrow 3)$-D-glucan from Poria cocos. Int Immunopharmacol. 2010; 10(4): 398-405.

[8] Zhao YY, Lei P, Chen DQ, Feng YL, Bai X. Renal metabolic profiling of early renal injury and renoprotective effects of Poria $\operatorname{cocos}$ epidermis using UPLC Q-TOF/HSMS/MS ${ }^{\mathrm{E}}$. J Pharm Biomed Anal. 2013; 81-82: 202-209.

[9] Baliga R, Ueda N, Walker PD, Shah SV. Oxidant mechanisms in toxic acute renal failure. Am J Kidney Dis. 1997; 29(3): 465-477.

[10] Han MH, Park C, Jin CY, et al. Apoptosis induction of human bladder cancer cells by sanguinarine through reactive oxygen species-mediated up-regulation of early growth response gene-1. PLoS One. 2013; 8(5): e63425.

[11] Leung PY, Miyashita K, Young M, Tsao CS. Cytotoxic effect of ascorbate and its derivatives on cultured malignant and nonmalignant cell lines. Anticancer Res. 1993; 13(2): 475-480.

[12] Prosperini A, Juan-Garcia A, Font G, Ruiz MJ. Reactive oxygen species involvement in apoptosis and mitochondrial damage in Caco- 2 cells induced by enniatins $A, A_{1}, B$ and $B_{1}$. Toxicol Lett. 2013; 222(1): 36-44.

[13] Shin HR, You BR, Park WH. PX-12-induced HeLa cell death is associated with oxidative stress and GSH depletion. Oncol Lett. 2013; 6(6): 1804-1810.

[14] Uetaki M, Tabata S, Nakasuka F, Soga T, Tomita M. Metabolomic alterations in human cancer cells by vitamin $\mathrm{C}$-induced oxidative stress. Sci Rep. 2015; 5: 13896.

[15] Dargel R. Lipid peroxidation: a common pathogenetic mechanism? Exp Toxic Pathol. 1992; 44(4): 169-181.

[16] Pelicano H, Martin DS, Xu RH, Huang P. Glycolysis inhibition for anticancer treatment. Oncogene. 2006; 25(34): 4633-4646. 
[17] Simons AL, Mattson DM, Dornfeld K, Spitz DR. Glucose deprivation-induced metabolic oxidative stress and cancer therapy. J Cancer Res Ther. 2009; 5(Suppl 1): S2-6.

[18] Miccoli L, Oudard S, Sureau F, Poirson F, Dutrillaux B, Poupon MF. Intracellular $\mathrm{pH}$ governs the subcellular distribution of hexokinase in a glioma cell line. Biochem J. 1996; 313(Pt 3): 957-962.

[19] Cheong JH, Park ES, Liang J, et al. Dual inhibition of tumor energy pathway by 2-deoxyglucose and metformin is effective against a broad spectrum of preclinical cancer models. Mol Cancer Ther. 2011; 10(12): 2350-2362.

[20] Priebe A, Tan L, Wahl H, et al. Glucose deprivation activates AMPK and induces cell death through modulation of Akt in ovarian cancer cells. Gynecol Oncol. 2011; 122(2): 389-395.
[21] Lee YK, Park OJ. Regulation of mutual inhibitory activities between AMPK and Akt with quercetin in MCF-7 breast cancer cells. Oncol Rep. 2010; 24(6): 1493-1497.

[22] Bolster DR, Crozier SJ, Kimball SR, Jefferson LS. AMP-activated protein kinase suppresses protein synthesis in rat skeletal muscle through down-regulated mammalian target of rapamycin (mTOR) signaling. J Biol Chem. 2002; 277(27): 23977-23980.

[23] Yip KW, Reed JC. Bcl-2 family proteins and cancer. Oncogene. 2008; 27(50): 6398-6406.

[24] Kuhajda FP. AMP-activated protein kinase and human cancer: cancer metabolism revisited. Int J Obes (Lond). 2008; 32(Suppl 4): S36-41.

[25] MacFarlane M, Robinson GL, Cain K. Glucose - a sweet way to die: metabolic switching modulates tumor cell death. Cell Cycle. 2012; 11(21): 3919-3925. 